\title{
Prävalenz von Antikörpern gegen Viren und Chlamydien bei Krankenhauspatienten mit und ohne koronarer Herzerkrankung
}

\author{
Prevalence of Antibodies Against Viruses and Chlamydia Among Hospitalized Patients \\ With or Without Coronary Heart Disease
}

\author{
S. Franck, H. F. Rabenau und H. W. Doerr
}

Zusammenfassung: Es wurde die Seroprävalenz von Erregern mit und ohne Tropismus zu Gefäßendothelzellen, wie Chlamydia (C.) pneumoniae, C. trachomatis, C. psittaci, verschiedene Herpesviren (Cytomegalie-Virus (CMV), Epstein-Barr-Virus (EBV), Herpes simplex Typ 1 und 2-Virus, Varizella Zoster-Virus (VZV)) sowie Masern- und Mumpsviren, bei Patienten mit koronarer Herzerkrankung $(K H K)(n=167)$ und zwei Kontrollkollektiven ohne Herzerkrankung ( $n=400, n=108)$ ermittelt. Die IgG-Antikörperprävalenzen betrugen im KHK-Kollektiv für C. pneumoniae $79,6 \%$ bzw. in den Kontrollkollektiven $72,5 \%$ und $66,7 \%$, für C. trachomatis $4,8 \%$ bzw. $6,8 \%$ und $2,8 \%$, für C. psittaci $3 \%$ bzw. $3,5 \%$ und $6,5 \%$, für $\mathrm{CMV}$ $72,9 \%$ bzw. $74,3 \%$ und $79,2 \%$, für EBV $95,1 \%$ bzw. $93,1 \%$ und $94 \%$, für Herpes simplex $1 / 291,8 \%$ bzw. $87.4 \%$ und $91,3 \%$, für Masern $99,2 \%$ bzw. $100 \%$ und für Mumps $93,4 \%$ bzw. $86,5 \%$ und $84,8 \%$. Die Prävalenzen der VZV-IgA waren $60,3 \%$ bzw. $57,3 \%$ und $54 \%$. Bei dieser Untersuchung zeigten sich somit keine signifikanten Unterschiede in den Antikörperprävalenzen zwischen den einzelnen Kollektiven. Wurden kranke, stationäre Patienten aus dem Kontrollkollektiv ausgeschlossen, so fand sich in diesem zweiten Kontrollkollektiv mittels $\mathrm{Chi}^{2}$-Test eine signifikant niedrigere Prävalenz $(66,7 \%)$ der C. pneumoniae-IgG-Antikörper im Vergleich zu Patienten mit KHK $(79,6 \%),(p=0,02)$. Die Untersuchung der geschlechtsspezifischen Prävalenzen zeigte für Männer (82,6\% bzw. $78,5 \%$ und $73,2 \%)$ eine höhere Durchseuchung als für Frauen $(55,6 \%$ bzw. $63 \%$ und $59,6 \%$ ). Beim Vergleich gleichgeschlechtlicher Gruppen fanden sich keine signifikanten Unterschiede. Eine Assoziation von C. pneumoniae oder CMV mit der atherosklerotischen, koronaren Herzerkrankung konnte somit durch unsere Untersuchung nicht bestätigt werden. Die Wahl der Kontroll-Gruppen kann möglicherweise die Ergebnisse der Metaanalyse früherer Assoziationsstudien beeinflußt haben.

Korrespondenzadresse: Dr. med. Susanne Franck, Institut für Medizinische Virologie, Paul-Ehrlich-Str. 40, D-60596 Frankfurt am Main.

Fax: ++49 (0) 69-6301-6477; E-mail: franck@em.uni-frankfurt.de Eingegangen: 2. Mai 2000/Angenommen: 21. Juni 2000
Schlüsselwörter: Chlamydien; Cytomegalie-Virus; Koronare Herzkrankheit; Atherosklerose; HerpesViren.

Summary: The seroprevalences of infectious agents with or without tropism for endothelial cells such as Chlamydia (C.) pneumoniae, C. trachomatis, C. psittaci, various Herpes viruses (Cytomegalovirus (CMV), Epstein - Barr virus, (EBV), Herpes simplex virus type 1 and 2, Varicella - Zoster virus (VZV)), measles and mumps virus in patients with coronary heart disease (CHD) $(n=167)$ were compared with those in two control groups without coronary heart disease $(n=400, n=108)$, one group including patients being hospitalized for other severe diseases. The seroprevalences of antibodies against C. pneumoniae were $79.6 \%$ in CHD patients vs. $72.5 \%$ and $66.7 \%$ for the two control groups. The respective prevalences of IgG antibodies against $C$. trachomatis were $4.8 \%$ vs. $6.8 \%$ and $2.8 \%$, against C. psittaci $3 \%$ versus $3.5 \%$ and $6.5 \%$, against CMV $72.9 \%$ versus $74.3 \%$ and 79.2 , against EBV $95.1 \%$ versus $93.1 \%$ and $94 \%$, against simplex $1 / 291.8 \%$ versus $87.4 \%$ and $91.3 \%$, against measles $99 \%$ versus $100 \%$ and against mumps $93.4 \%$ versus $86.5 \%$ and $84.8 \%$. The seroprevalences for VZV-IgA were $60.3 \%$ versus $57.3 \%$ and $54 \%$. There were hence no significant differences in the antibody seroprevalences between the different groups. Excluding the hospitalized patients from the control group, the prevalence of $\mathrm{C}$. pneumoniae - IgG antibodies was significantly lower in controls $(66.7 \%)$ than in patients with CHD (79.6\%). (Chi'-test $\mathrm{p}=0,02)$. However, men had higher prevalences $(82.6 \%$ in patients, $78.5 \%$ and $73.2 \%$ in controls) than women $(55.6 \%$ versus $63 \%$ and $59.6 \%$ ). After adjustment for gender, the difference between cases and controls was therefore no longer significant. Our study thus did not confirm an association between C. pneumoniae or Cytomegalovirus and coronary heart disease. The choice of the control group may have strongly influenced the outcomes of a metaanalysis of former studies.

Keywords: chlamydia; coronary heart disease; atherosclerosis; Cytomegalovirus; Herpes virus. 
$\mathbf{S}$ eit Rudolf Virchow wird für die Atherosklerose eine entzündliche, möglicherweise infektbedingte Ursache vermutet. In den letzten Jahren wurde zunächst das Cytomegalievirus (CMV) $[1,9,11,12$, 23. 24, 25], später Chlamydia pneumoniae als ätiologisches Agens der Atherosklerose benannt [8, 9, 11, $12,14,15,26,43]$. Unterstützt werden diese Daten durch entsprechende epidemiologische Untersuchungen $[1,14,30,34]$, direkte Erreger-Nachweise in den betroffenen Gefäßen (mittels Polymerase Kettenreaktion und Anzucht auf Zellkulturen) [21], sowie durch in vivo-Versuche an Ratten [26, 33]. Neuere Studien haben jedoch auch kritische Einwände und Alternativvorstellungen gegen einen kausalen Zusammenhang vorgebracht, wonach $C$. pneumoniae oder CMV eher Kofaktor als Auslöser der Atherosklerose ist.

Infektionsserologische Untersuchungen werden bei Krankenhauspatienten häufig aus differentialdiagnostischen Gründen im Sinne einer Ausschlußdiagnose durchgeführt. Eine ausreichende Fallzahl vorausgesetzt, ergibt sich daraus die Möglichkeit zu seroepidemiologischen Erhebungen bei Personen mit und ohne spezifisches Infektionsrisiko.

Um die o.g. Hypothese zu überprüfen, wurden umfangreiche retrospektive Analysen der infektionsserologischen Daten vorgenommen, die routinemäßig im Rahmen der mittelbaren Krankenversorgung der Frankfurter Universitätsklinik und Umgebung zur Abklärung verschiedener Virus- und Chlamydieninfektionen erhobenen worden waren. Als Träger einer manifesten oder beginnenden Atherosklerose werden Patienten eingestuft, die wegen koronarer Herzbeschwerden von ihren Ärzten zur Untersuchung in das Krankenhaus eingewiesen worden sind. Als Vergleichsgruppe dienten Patienten, die aufgrund anderer Ursachen im Krankenhaus untersucht wurden.

Im Rahmen der vorliegenden Studie wurden bei 167 Patienten mit der klinischen Diagnose KHK Untersuchungen auf Antikörper gegen C. pneumoniae durchgeführt und retrospektiv ausgewertet. Zusätzlich wurden Antikörper gegen C. trachomatis, C. psittaci sowie verschiedene virale Erreger (CMV, Epstein Barr Virus (EBV), Herpes simplex Virus 1 und 2 (HSV 1/2), Varizella Zoster Virus (VZV), Masern und Mumpsvirus) bestimmt, um so Hinweise auf eventuelle Assoziationen dieser Infektionserreger mit Atherosklerose zu erhalten.

\section{Patienten und Methoden}

Im Zeitraum August 1997 bis Oktober 1999 wurden Patientenseren von verschiedenen Einsendern des Universitätsklinikums Frankfurt am Main, sowie von externen Krankenhäusern aus dem Rhein-Main-Gebiet untersucht und retrospektiv ausgewertet. Hierzu wurden drei Kollektivgruppen gebildet:

Gruppe 1: Patienten (überwiegend aus der kardiologischen Ambulanz) mit der klinischen Diagnose „koronare Herzerkrankung" (KHK), die mittels EKG bestätigt wurde, $(n=167)$,

Gruppe 2: Patienten ohne KHK $(n=400)$,

Gruppe 3: = Gruppe 2 unter Ausschluß internistischer, stationärer Patienten $(\mathrm{n}=108)$.

Grundsätzlich wurden nur Patienten älter als 40 Jahre in die Studie aufgenommen. Die Gruppengröße variiert zum Teil geringfügig, da nicht immer ausreichend Serummaterial zur Durchführung aller Testparameter zur Verfügung stand (siehe Tabelle 1).

Für die serologischen Analysen wurden folgende Tests, entsprechend den Herstellerangaben, verwendet:

Nachweis von C. pneumoniae-IgG, C. trachomatisIgG, C. psittaci-IgG (SeroFIA Mikroimmunfluoreszenz-Assay; Hain Diagnostika, Nehren), CMV-IgG (Enzygnost Anti-CMV-IgG-ELISA, Behring Diagnostics GmbH, Marburg), EBV EBNA 1-IgG (anti-EBV recombinant EBNA-IgG-ELISA, Biotest Diagnostics Corporation, Dreieich), HSV 1/2-IgG (Herpes Simplex Virus $1+2$ partiell gereinigtes IgG-ELISA, Gull Laboratories GmbH, Bad Homburg), VZV-IgA (als Reaktivierungsmarker) (Enzygnost Anti-VZV-IgA-ELISA. Dade Behring, Marburg), Masern-IgG (Enzygnost Anti-Masern-IgG-ELISA, Dade Behring, Marburg) und Mumps-IgG (Enzygnost Anti-Mumps-IgGELISA, Dade Behring, Marburg). Die Nachweise virusspezifischer Antikörper wurden mittels ELISA durchgeführt, während die Untersuchung auf Chlamydien-spezifische-Antikörper mittels Mikroimmunfluoreszenz-(MIF-)Test erfolgte.

Zur Berechnung der Signifikanzen wurde der $\mathrm{Chi}^{2}-$ Test mit Yates-Korrektur angewendet und das 95\%Konfidenzintervall ermittelt.

Tabelle 1 Anzahl männlicher und weiblicher-Patienten, die in die Studie aufgenommen wurden

\begin{tabular}{|c|c|c|c|c|c|c|c|c|c|}
\hline & \multicolumn{3}{|c|}{$\begin{array}{l}\text { KHK-Patienten } \\
\text { Gruppe } 1\end{array}$} & \multicolumn{3}{|c|}{$\begin{array}{c}\text { Kontrollkollektiv } 1 \\
\text { Gruppe } 2\end{array}$} & \multicolumn{3}{|c|}{$\begin{array}{c}\text { Kontrollkollektiv } 2 \\
\text { Gruppe } 3\end{array}$} \\
\hline & Anzahl & Anteil (\%) & Altersmedian & Anzahl & Anteil (\%) & Altersmedian & Anzahl & Anteil (\%) & Altersmedian \\
\hline Männer & 149 & 89,2 & 59 & 246 & 61,5 & $57^{\prime}$ & 56 & 51,9 & 57 \\
\hline Frauen & 18 & 10,8 & 58 & 154 & 38,5 & 55 & 52 & 48,1 & 51 \\
\hline
\end{tabular}




\section{Ergebnisse}

Die Analyse der Antikörperprävalenzen crfolgte in den einzelnen Gruppen sowohl geschlechtsspezifisch als auch geschlechisunabhängig.

Bei 133 der 167 Patienten (79,6\%) mit koronarer Herzerkrankung (123 der 149 männlichen $[82,6 \%]$ und 10) der 18 weiblichen $[55,6 \%]$ Patienten) wurden $C$. pneumoniae spezifische IgG-Antikörper nachgewiesen. Während im Kontrollkollektiv - Gruppe 2 - die Antikörperprävalenz mit $72.5 \%$ (290/400; 193 von 246 männlichen $[78,5 \%]$ und 97 von 154 weiblichen $163 \%$ ] Patienten) geringfügig und statistisch nicht signifikant niedriger lag $(p=0,094)$, zeigte das zweite Kontrollkollektiv - Gruppe 3 - mit 66,7\% (72/108; 41 von 56 männlichen $[73,2 \%]$ und 31 von 52 weiblichen [59.6\% | Patienten) einen statistisch signifikanten Unterschied zu Gruppe $1(p=0,023)$. Ein Vergleich der Durchseuchung von Männern und Frauen zeigt geschlechtsspezifische, signifikante Unterschiede der Antikörperprävalenz in Gruppe $1(p=0,017)$ und Gruppe $2(p=0,001)$, nicht jedoch in Gruppe $3(p=0,196)$ (siehe Abb. 1-3).
Entsprechend der Durchseuchungsrate mit C. trachomatis in der Gesamtbevölkerung zeigten $4,8 \%$ der KHK-Patienten $(8 / 167 ; 5.4 \%$ der männlichen und keine der weiblichen Patienten); 6.8\% der Gruppe 2 (27/400: $6,5 \%$ der männlichen und $7,1 \%$ der weiblichen Patienten) und $2.8 \%$ der Gruppe $3(3 / 108 ; 3.6 \%$ der männlichen und $1,9 \%$ der weiblichen Patienten) spezifische Antikörper. Eine ebenfalls niedrige Prävalenz wurde für C. psittaci-IgG nachgewiesen (Gruppe 1: 3\% [Männer 3,4\%; Frauen 0\%]; Gruppe 2: 3,5\% [Männer 4,5\%; Frauen 2\%] und Gruppe 3: 6,5\% [Männer 3,6; Frauen 9,6\%]).

CMV-IgG spezifische Antikörper wurden bei $72,9 \%$ der KHK-Patienten $(73,1 \%$ männlichen und $72 \%$ bei weiblichen Patienten) nachgewiesen. In der Gruppe 2 waren $74,3 \%$ der Patienten Antikörper-positiv (Männer $74,6 \%$; Frauen $73,9 \%$ ) und in Gruppe 3 bei $79.2 \%$ (Männer 83,3\%; Frauen 71,4\%). Die geschlechtsspezifischen Unterschiede in der CMV-Antikörperprävalenz waren in allen drei Gruppen nicht signifikant $(p=0,9$; $\mathrm{p}=0,98 ; \mathrm{p}=0,252$ ).

Zum Nachweis einer durchgemachten Epstein-BarrVirus Infektion wurde EBV-EBNA-1 IgG bestimmt.
Abbildung 1 Antikörperprävalenz verschiedener Erreger bei KHK-Patienten (Gruppe 1) und zwei Kontrollkollektiven (Gruppe 2: [internistische] Patienten ohne KHK, Gruppe 3: Patienten ohne KHK unter Ausschluß internistischer Patienten) mit 95\%-Konfidenzintervall
Antikörperprävalenz von Frauen und Männner

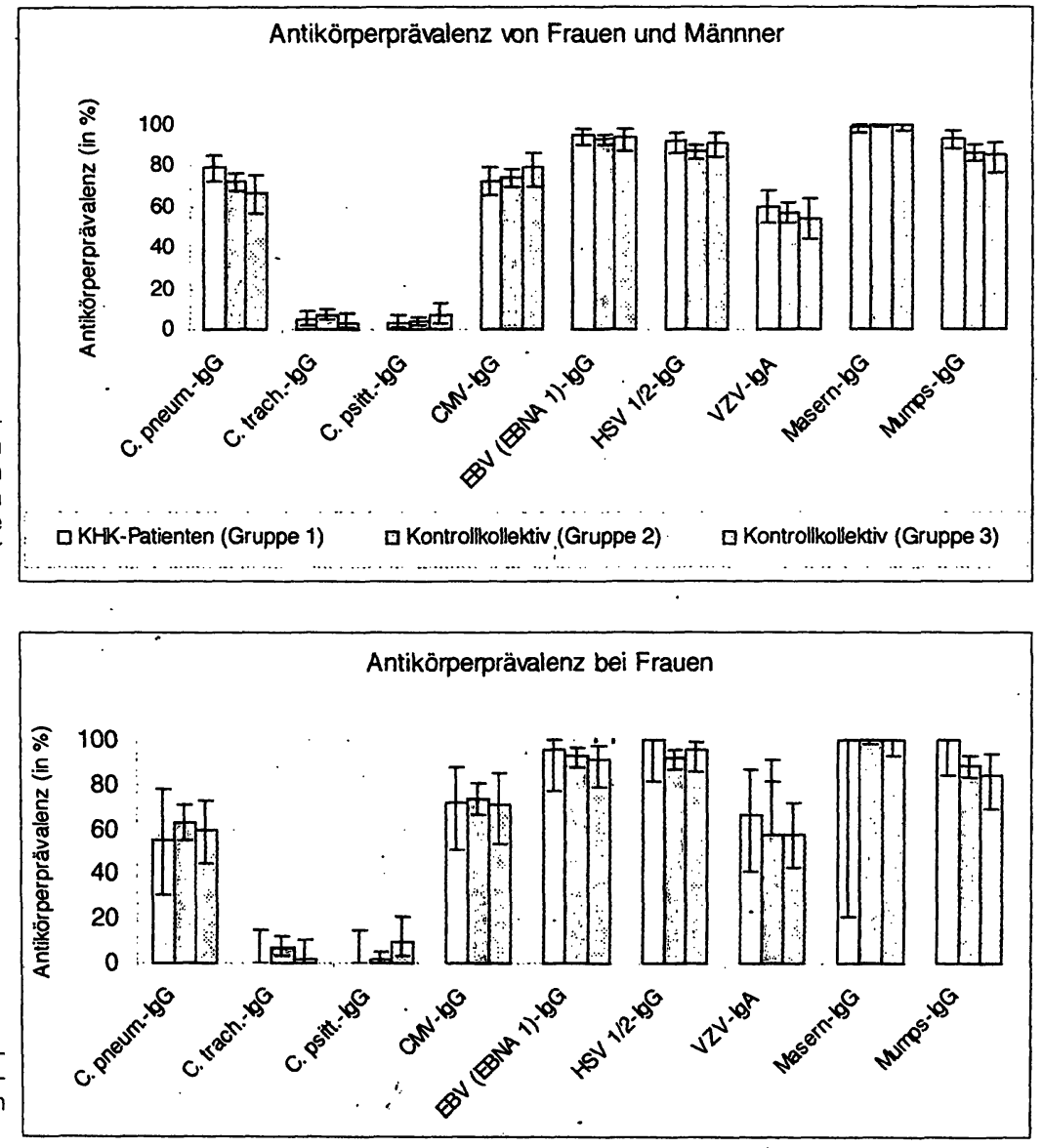

口KHK-Patienten (Gruppe 1) $\square$ Kontrollkollektiv (Gruppe 2) $\quad \square$ Kontrollkollektiv (Gruppe 3)

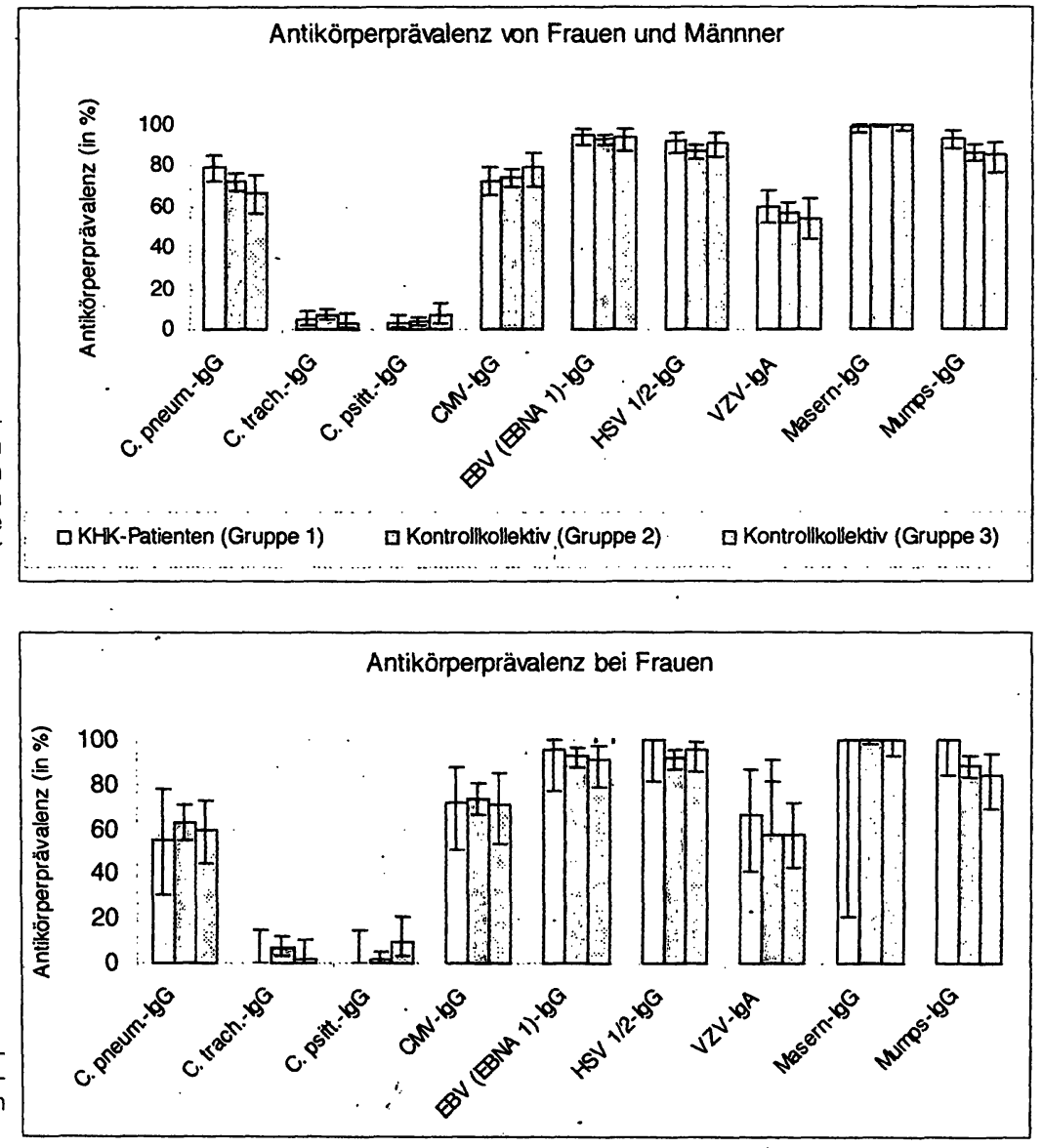

Abbildung 2 Antikörperprävalenz verschiedener Erreger bei weiblichen $\mathrm{Pa}$ tienten (Angaben zu den Kollektiven siehe Abb. 1) 
Abbildung 3 Antikörperprävalenz verschiedener Erreger bei männlichen Patienten (Angaben zu den Kollektiven siehe Abb. 1)

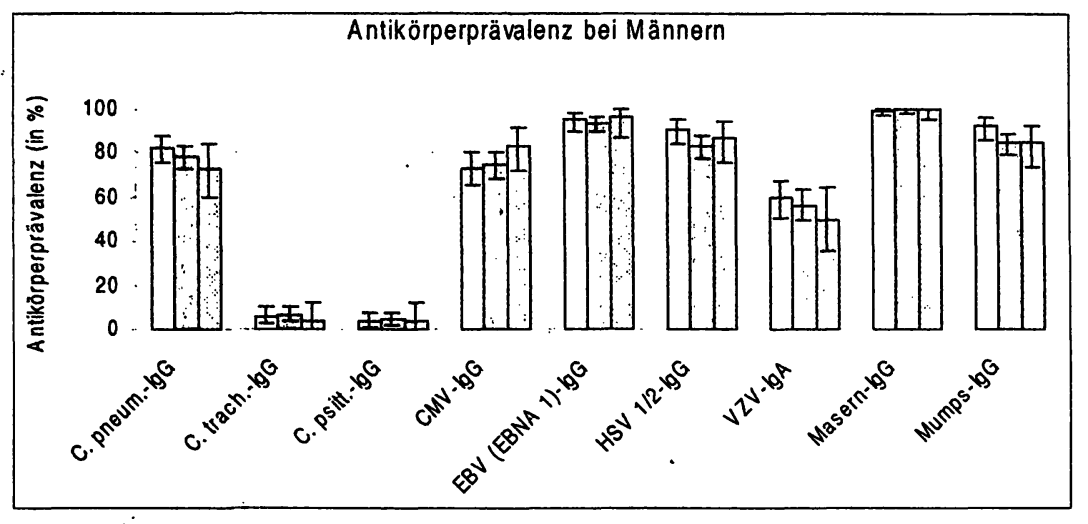

Hier betrug die Durchseuchung in allen 3 Gruppen ca. 94\%. Bei KHK-Patienten zeigten 95,1\% (95\% Männer; $95,5 \%$ Frauen) spezifische Antikörper, in Gruppe 2 93,1\% (Männer 93,5\%; Frauen 92,6\%) und in Gruppe $394,0 \%$ (Männer 96,3\%; Frauen $91,3 \%$ ).

Eine ähnlich hohe Durchseuchung wurde auch bei HSV Typ 1 und 2 ermittelt. Sie liegt - ebenfalls in allen 3 Gruppen - bei ca. $90 \%$, bei KHK-Patienten $91,8 \%$ (Männer $90,8 \%$; Frauen $100 \%$ ); in Gruppe 2 $87,4 \%$ (Männer $83,1 \%$; Frauen $92,2 \%$ ) und in Gruppe 3 91,3\% (Männer 87,3\%; Frauen 95,8\%). In Gruppe 2 traten geschlechtsspezifische signifikante Unterschiede in der Durchseuchung mit Herpes simplex Viren auf $(p=0,012)$, nicht jedoch in Gruppe $1(p=0,465)$ und Gruppe $3(p=0,252)$.

Die Patientenseren wurden auf VZV-IgA untersucht, um VZV-Reaktivierungen und damit den möglichen Einfluß von Immunsuppression und Entzündungen auf koronare Gefäßerkrankung zu erfassen. Auch hier zeigten sich keine signifikanten Unterschiede in den Antikörperprävalenzen zwischen den drei Kollektiven (60,3\% [Männer 59,4\%; Frauen 66,7\%] bei KHK-Patienten; in Gruppe $257,3 \%$ [Männer 56,4\%; Frauen $58,15 \%$ ] und in Gruppe $354,0 \%$ [Männer 50\%; Frauen $58 \%]$ ).

Ebenfalls keine signifikanten Differenzen traten bei der Antikörperprävalenz gegenüber Mumps und Masern auf. Die Masern-IgG Durchseuchung betrug in allen drei Gruppen nahezu $100 \%(99,2 \%$ bei den männlichen KHK-Patienten, $100 \%$ bei den restlichen Gruppen).

Die Prävalenzen für Mumps-IgG betrugen im KHKKollektiv 93,4\% (Männer 92,5\%; Frauen 100\%), in Gruppe $282,6 \%$ (Männer 84,7\%; Frauen $89 \%$ ) und in Gruppe $384,8 \%$ (Männer 85\%; Frauen 84,6\%).

Die Untersuchung der Antikörper-Prävalenz zeigt bei Trennung nach Geschlecht für einzelne Erreger deutliche Unterschiede, vor allem für $\mathrm{C}$. pneumoniae in Gruppe 1 und 2, sowie für Herpes simplex Viren in Gruppe 2.

Eine altersdifferenzierte Betrachtung (Gruppe 40$49,50-59$ und älter als 60 Jahre) zeigt keine signifikanten Unterschiede.

\section{Diskussion}

In zahlreichen Studien wurde auf einen möglichen kausalen Zusammenhang zwischen Chlamydia pneumoniae Infektionen und koronarer Herzerkrankung (KHK) hingewiesen $[1,8,9,11,12,14,15,21,26,30$, 34, 43]. Obwohl Experimente am Rattenmodell diese Kausalität untermauern, stehen weitere Erreger, wie z.B. das CMV, in Verdacht, als Kofaktor oder Ursache eine tragende Rolle bei der Entstehung der KHK einzunehmen [1, 9, 11, 23, 24, 25]. Als pathogenetischer Wirkmechanismus wird über die Stimulation von TZellen - als Folge einer chronischen Infektion durch Viren, Bakterien oder gar Impfungen - spekuliert. Diese Stimulation könnte zur Sezernierung von Zytokinen und zur Proliferation des Gefäßendothels und der glatten Gefäßmuskulatur führen und somit die Atherosklerose begünstigen [32]. Diese Stimulation muß nicht spezifisch durch C. pneumoniae erfolgen. sondern ist vermutlich als multifaktorielles Geschehen zu bewerten, bei der neben einer Chlamydieninfektion noch andere Erreger und Risikofaktoren beteiligt sind.

Danesh et al. haben eine Reihe von seroepidemiologischen Studien zur Assoziation von C. pneumoniae und Atherosklerose einer kritischen Metaanalyse unterworfen und die darin gemachten Aussagen einer positiven Korrelation in Frage gestellt [9]. Auch in unseren Auswertungen zeigt sich, daß die Auswahl der „richtigen“ Vergleichsgruppe entscheidend für die seroepidemiologische Aussage ist: Vor dem Hintergrund der C. pneumoniae-Durchseuchung von Kranken, die sich wegen einer anderēn Krankheit in ärztliche Behandlung gegeben haben, ist die Prävalenz von C. pneumoniae-Antikörpern bei Patienten, die wegen einer Koronarerkrankung zur Untersuchung kommen, nicht erhöht.

In der vorliegenden retrospektiven Studie wurden die Antikörperprävalenzen gegenüber verschiedenen viralen Erregern (CMV, EBV, HSV 1/2, VZV, Masern, Mumpsvirus) sowie $C$. pneumoniae, C. trachomatis und C. psittaci bei Patienten mit KHK sowie zwei Kontrollkollektiven ermittelt. Diese Erreger wurden ausgewählt, da sie zum großen Teil persistente Infek- 
tionen verursachen oder als Auslöser von Exanthemen cndothelotrop sind (Masern). Als Kontrolle dienten Erreger ohne Gefäßtropismus, wie Mumpsviren.

Zur Aufdeckung von möglichen Einflußfaktoren auf eine koromare Herzerkrankung wic Immunsuppression und chronische Entzündung wurden VZV-IgA Antikörper als Reaktivicrungsmarker untersucht, denn VZV zeigt in der Bevölkerung eine nahezu 100\%ige Durchseuchung.

Un statistisch relevante Aussagen treffen zu können, ist die Auswahl des Kontrollkollektivs von wesentlichem Einfluß. Eine KHK gewinnt erst im mittleren Lebensalter an Bedeutung, daher wurden Patientenproben aus jüngerem Lebensalter ( $<40$ Jahre) nicht einbezogen. Es zeigt sich in den verschiedenen Altersgruppen (40-49, 50-59 und älter 60 Jahre) kein signifikanter Anstieg, so daß davon ausgegangen werden kann, daß cinc Infektion mit den untersuchten Erregern in den meisten Fällen bis zum 40. Lebensjahr erfolgt ist.

Erwartungsgemäß zeigt sich in der KHK-Gruppe eine Dominanz an männlichen $(89 \%)$ gegenüber weiblichen (11\%) Patienten (siehe Tabelle 1), die bei der Bewertung der Gesamtprävalenz zu berücksichtigen ist.

Unsere Daten zeigen, daß die Durchseuchung mit Mumps und Masern sowie für die verschiedenen Viren der Herpesgruppe (HSV 1/2, EBV, VZV [- IgA]) in dem KHK-Kollektiv (Gruppe 1) und den beiden Kontrollkollektiven etwa gleich hoch ist. Die Prävalenz liegt für Masern-IgG bei nahezu $100 \%$, für MumpsIgG bei etwa 93\% in Gruppe 1, 83\% in Gruppe 2 bzw. $85 \%$ in Gruppe 3, für EBV-IgG bei $95 \%$ in Gruppe 1, $93 \%$ in Gruppe 2 bzw. 94\% in Gruppe 3 und für VZVIgA bei $60 \%$ in Gruppe $1,57 \%$ in Gruppe 2 bzw. $54 \%$ in Gruppe 3. Die Unterschiede zwischen den einzelnen Gruppen sind im $\mathrm{Chi}^{2}$-Test nicht signifikant ( $\left.\mathrm{p}>0,05\right)$.

Für HSV 1/2-IgG läßt sich eine Antikörper-Prävalenz von $92 \%$ in Gruppe 1,87\% in Gruppe 2 bzw. 91\% in Gruppe 3 nachweisen. Bei Betrachtung der geschlechtsspezifischen Differenzen fällt auf, daß in den Gruppen 1, 2 und 3 die weiblichen Patienten eine um ca. 10\% höhere Durchseuchung aufweisen als Männer. Der Unterschied ist statistisch signifikant in Gruppe 2 $(p=0,012)$. Für HSV 2 sind solche geschlechtspezifischen Unterschiede in ähnlichen Ausmaß bereits vielfach beschrieben $(2,6,10)$.

Besonderes Augenmerk richtet sich in der wissenschaftlichen Literatur auf die Rolle des Cytomegalievirus bei der Manifestation der Atherosklerose [1, 11, 13, 17, 23, 24, 25, 27, 32]. Eine entsprechende Assoziation wird durch unsere Daten jedoch nicht bestätigt. So lassen sich keiǹe signifikanten Unterschiede zwischen den drei Kollektiven feststellen (CMV-IgGPrävalenz bei KHK-Patienten $72,9 \%$, in Gruppe 2 $74,3 \%$ und in Gruppe 3.74,2\%).

Im Gegensatz zu diesen hohen Prävalenzen waren Antikörper gegen die Erreger $C$. trachomatis und $C$. psittaci in allen drei Patientengruppen nur selten nachweisbar (C. trachomatis-IgG-Positivität: 4,8-6,8\%, C. psittaci-IgG-Positivität: 3-6,5\%). Die Unterschiede sind statistisch nicht signifikant.
Anders verhält es sich bej C. pneumoniae. Während ein Vergleich zwischen dem KHK-Kollektiv (Prävalenz 79,6\%) und der Gruppe 2 (internistische, stationäre Patienten ohne KHK-Diagnose - 72.5\%) mittels $\mathrm{Chi}^{2}$-Test keinen signifikanten Unterschied zeigt $(p=0,09)$, ist ein solcher gegenüber Gruppe 3 (Patienten ohne KHK, unter Ausschluß internistischer, stationärer Patienten - 66,7\%) festzustellen. Die Betrachtung der Konfidenzintervalle läßt zunächst einen Unterschied nicht offensichtlich werden, erst die Überprüfung mittels $\mathrm{Chi}^{2}$-Test und Yates-Korrektur ( $\mathrm{p}=$ 0,023 ) weist darauf hin.

Um diesen Unterschied näher zu untersuchen, wurden die Patienten nach Geschlecht und Alter differenziert. Der Vergleich zwischen den männlichen $\mathrm{KHK}$ Patienten mit den männlichen Patienten der ersten und zweiten Kontrollgruppe ergab keine signifikanten Unterschiede der C. pneumoniae Prävalenz. Ebenso ergab der Vergleich zwischen den KHK-Patientinnen mit den Patientinnen der ersten und zweiten Kontrollgruppe keinen signifikanten Unterschied. Demgegenüber wurde jedoch ein signifikanter Unterschied zwischen männlichen und weiblichen Patienten innerhalb der jeweiligen Gruppe 1 bzw. Gruppe 2 ermittelt (Gruppe 1: $\mathrm{p}=0,017$, Gruppe 2: $\mathrm{p}=0,001)$. In der Gruppe 3 ist kein signifikanter Unterschied zwischen den geschlechtsspezifischen Prävalenzen $z u$ verzeichnen $(p=0,196)$. Die zunächst scheinbar signifikant höhere geschlechtsunabhängige Prävalenz von $C$. pneumoniae in der KHK-Gruppe gegenüber dem zweiten Kontrollkollektiv (Gruppe 3) kann durch das Überwiegen von männlichen Patienten in der KHK-Gruppe ( $89 \%$ versus $52 \%$ ) erklärt werden.

Wodurch.sich diese geschlechtsspezifischen Unterschiede ergeben, ist bisher noch nicht ergründet. In der Literatur findet man von Saikku [35] und Coles [7] ebenfalls die Beschreibung einer höheren AntikörperPrävalenz bei Männern gegenüber Frauen. Solche geschlechtsspezifischen Unterschiede sind unter anderem auch bei Herpes simplex Virus Typ 2 bekannt [2, 6 , 10] und konnten durch unsere Daten erneut bestätigt werden. Der Übertragungsweg von HSV - 2 erfolgt durch die Genitalschleimhaut, während C. pneumoniae eine respiratorische Übertragung hat. Möglicherweise könnte Rauchen oder ein größeres Lungenvolumen bei Männern eine Aufnahme des Erregers begünstigen.

Unsere Ergebnisse verdeutlichen, daß die Auswahl des Kontrollkollektivs erheblich die Aussage und Qualität solcher seroepidemiologischen Studien beeinflußt [9]. Bei schwerkranken Patienten mit hoher C. pneumoniae Durchseuchung (Gruppe 2) mag die klinische Manifestation einer koronaren Herzerkrankung einer anderen Grunderkrankung untergeordnet sein, so daß eine Assoziation zwischen einer Chlamydieninfektion - im zeitlichen Verlauf - noch nicht zu treffen ist. Studien zur Prävalenz von Antikörpern können die Frage nach einer Assoziation der KḦK und einer infektiösen Ätiologie also nicht hinreichend beantworten. Verantwortlich hierfür ist u.a., daß Antikörper auf eine durchgemachte Infektion hinweisen und fast lebenslang per- 
sistieren. Reinfektionen oder Rezidive sind sowohl für Chlamydien als auch für etliche der untersuchten Erreger beschrieben, werden aber serologisch nur bedingt durch Titeranstieg erfaßt und können in einer epidemiologischen.Untersuchung nicht erkannt werden. Zur Klärung der o.g. Frage sind daher weitere Untersuchungen - vorzugsweise mit Erregerdirektnachweisen - erforderlich.

\section{Literatur}

1. Adam E, Melnick JL, Probtsfield JL, Petrie BL, Burek J, Bailey KR, McCollum MC CH. Bailey KR, DeBakey ME. High levels of Cytomegalovirus antibody in patients requiring vascular surgery for atherosclerosis. Lancet 1987;291-3.

2. Bahrdt B, Rabenau H, Weber B, Eibner J, Doerr HW. Prävalenz Herpes-simplex-Virus-Typ-2-spezifischer Antikörper bei Personen mit unterschiedlichen Infektionsrisiko. Z Hautk. 1992;67 (1):56-8. 3. Bauriedel G, Andrié R, Likungu JA, Welz A, Braun P, Lüderitz B. Persistence of Chlamydia pneumoniae in coronary plaque tissue: a contribution to the infection and immune hypothesis concerning unstable angina. Dtsch Med Wschr 1999;124:1408-13.

4. Carlisle SS, Nahata MC. Chlamydia pneumoniae and coronary heart disease. Ann Pharmacother 1999;33 (5):615-22.

5. Cellesi C, Sansoni A, Casini S, Migliorini L, Zacchini F, Gasparini R. Montomoli E, Bonacci A, Bravi A. Chlamydia pneuominiae antibodies and angiographically demonstrated coronary artery disease in a sample population from Italy. Atherosclerosis 1999;145 (1):81-5.

6. Chenot JF, Rabenau HF, Doerr HW. Virologie, Epidemiologie und Diagnostik des Herpes genitalis. Dtsch. Med. Wschr. 1999;124:158-62.

7. Coles KA, Plant AJ, Rile TV, Smith DW, Mc Quillan BM, Thompson PL. Lack of association between seropositivity to Chlamydia pneumoniae and carotid atherosclerosis. Am J Cardiol 1999;84 (7):825-8.

8. Cunningham MJ. Pasternak RC. The potential role of viruses in the pathogenesis of atherosclerosis. Circulation 1988; Vol 77, No. 7:964-6.

9. Danesh J, Collins R, Peto R. Chronic infections and coronary heart disease: is there a link? Lancet 1997;350:430-6.

10. Doerr HW, Rabenau HF. Dermatotrope Herpesviren, Infektionsbiologie, Epidemiologie und Diagnostik. Chemotherapie Jounal 1996;1:1-11.

11. Epstein SE, Speir E, Zhou YF, Leon M, Finkel T. The role of infection in restenosis and atherosclerosis: focus on cytomegalovirus. Lancet 1996;348:13-17.

12. Gaur LK, Peeling RW, Cheang M, Kimani J. Association of Chlamydia trachomatis Heat-Shock Protein 60 Antibody and HLA Class II DQ Alleles. J Infect Dis 1999;180:234-7.

13. Gulizia JM, Kandolf R, Kendal TJ, Thieszen SL, Wilson JE, Radio SJ. Costsanzo MR, Winters GL, Miller LL, McManus BM. Infrequency of Cytomegalovirus Genome in Coronary Arteriopathy of Human Heart Allografts. Am J Path. 1997;147( 2):461-75.

14. Gupta S. Leatham EW, Carrington D, Mendall MA, Kaski JC, Camm AJ. Elevated Chlamydia pneumoniae Antibodies, Cardiovascular Events, and Azithromycin in Male Survivors of Myocardial Infarction. Circulation 1997;96(2):404-7.

15. Gurfinkel E, Bozovich G, Daroca A, Beck E, Mautner B. Randomised trial of roxithoromycin in non-q-wave coronary syndromes: ROXIS pilot study. Lancet 1997;350:404-7.

16. Gurfinkel E, Bozovich G: Chlamydia pneumoniae: inflammation and instability of the atherosclerotic plaque. Atherosclerosis 1998;140 (1):17-9.

17. Hendrix MGR, Salimans MMM, van Boven CPA, Bruggeman CA. High Prevalence of Latently Present Cytomegalovirus in Arterial Walls of Patients suffering form Grade III Atherosclerosis. Am J Pathol 1990:136 (1):23-8.

18. Lamb DJ, Ferns GAA. Infection, immunisation and atherosclerosis: is there a link ? Vaccine 1999;17:559-64.

19. Leinonen M, Saikku P. Interaction of Chlamydia pneumoniae infection with other risk factors of atherosclerosis. Am Heart $J$ 1999:138 (5 Pt 2):504-6.
20. Lindholt JS, Fasting H, Henneberg EW, Ostergaard L. A review of Chlamydia pneumoniae and atherosclerosis. Eur J Vasc Endovasc Surg 1999:17 (4):183-9.

21. Maas M, Bartels C. Krüger S, Krause E, Engel PM, Dalhoff K. Endovascular presence of Chlamydia pneumoniae DNA is a generalized phenomenon in athersclerotic vascular diseasc. Atherosclerosis 1998;140(1):25-30.

22. Martinez MA, Kugan R, Silva JJ, Pinto ME, Vidal C, Huppo $H$. Seroprevalence of Chlamydia pneumoniae in Chile. Scand $J$ Infect Dis 1999;31 (1):103-4.

23. Melnick JL, Adam E. DeBakey ME. Possible Role of Cytomegalovirus in Atherogenesis. JAMA 1990;263 (16):2204-7.

24. Melnick JL, Dreesman GR, McCollum CH, Petrie BL, Burek J, DeBakey ME. Cytomegalovirus Antigen within Human Arterial Smooth muscle Cells. Lancet 1983:644-7.

25. Melnick JL, Hu C, Burek J, Adam E, DeBakey ME. Cytomegalovirus DNA in Arterial Walls of Patients with Atherosclerosis. $J$ Med Vir 1994;42:170-4.

26. Moazed TC., Campbell LA., Rosenfeld ME, Grastone JC, Kuo CC. Chlamydia pneumoniae Infection accelerates the progression of atherosclerosis in apolioprotein E-deficient mice. J Infect Dis 1999; 180:238-41.

27. Nieto FJ, Adam E, Sorlie P, Farzadegan H. Melnick JL, Comstock GW, Szklo M. Cohort Study of Cytomegalovirus Infection as a Risk Factor for Carotid Intimal-Medial Thickening, a Measure of Subclinical Atherosclerosis. Circulation 1996;96(5):922-7.

28. Noll G. Pathogenesis of atherosclerosis: a possible relation to infection. Atherosclerosis 1998;140(1):3-9.

29. Ochsendorf FR, Özdemir K, Rabenau HF, Fenner Th, Oremek R, Milbradt R, Doerr HW. Chlamydia trachomatis and male infertility: Chlamydia-IgA antibodies in seminal plasma are C. trachomatis specific and associated with an inflammatory response. JEADV 1999. 12:143-52.

30. Orfila JJ. Seroepidemiological evidence for an association between Chlamydia pneumoniae and atherosclerosis. Atherosclerosis 1998; 140(1):11-15.

31. Rabenau HF. Eibner B, Weber B, Bahrdt B, Doerr HW. Detection of Herpes simplex virus (HSV) type specific antibodies by a mircrotechnique western blot assay. Lab Med 1992;16:327-33.

32. Rothenbacher D, Hoffmeister A, Bode G, Wanner P, Koenig, W, Brenner H. Cytomegalovirus Infection and Coronary Heart Disease: Results of a German Case-Control Study. J Inf Dis 1999;179:690-2.

33. Saikku P, Laitinen K, Leinonen M. Animal models for Chlamydia pneumoniae infection. Atherosclerosis 1998;140(1):17-9.

34. Saikku P. The epidemiology and significance of Chlamydia pneumoniae. J Inf 1992;25(1):27-34.

35. Saikku P. Epidemiology of Chlamydia pneumoniae in atherosclerosis. Am Heart J 1999;138:500-3.

36. Schieferstein C. Typenspezifische Herpes simplex Virus-Antikörper: Vergleich verschiedener ELISA-Testsysteme und eines Westernblot. Lab Med 1997;21 (29):107-8.

37. Schussheim A, Fuster V. Antibiotics for myocardial infarction? A possible role of infection in atherogenesis and acute coronary syndromes. Drugs 1999:57 (3):283-91.

38. Sessa R, Di Pietro M, Santino I, del Piano M, Varveri A, Dagiant $A$, Penco $M$. Chlamydia pneumoniae infection and atherosclerotic coronary disease. Am Heart J 1999:137 (6):1116-9.

39. Sorlie, Adam E, Menick S, Fosom A, Skelton T. Chambless LE, Barnes R, Melnick JL et al. Cytomegalovirus/Herpesvirus and Carotid Atherosclerosis: The ARIC Study. J Med Vir 1994;42:33-7.

40. Stille W, Dittmann R. Arteriosklerose als Folge einer chronischen Infektion durch Chlamydia pneumoniae. Herz 1998;23:18592.

41. Strachan D P, Carrington D, Mendall M A, Ballam L, Morris J, Kbutland B, Sweetnam P M, Elwood P C. Relation of Chlamydia pneumoniae serology to mortality and incidence of ischaemic heart disease over 13 years in the Caerphilly prospective heart disease study. BMJ 1999;318:1035-40.

42. Taylor-Robinson D. Chlamydia pneumoniae in vascular tissue. Atherosclerosis 1998;140(1):21-4.

43. Tiran A, Tio RA, Osswaarde JM, Tiran B, den Heijer P. The TH, Wilders-Truschnig M. Coronary Angioplasty induces rise in Chlamydia pneumoniae-specific antibodies. J Clin Microbiol 1999;1013-7.

44. Visser MR, Vercellotti GM. Herpes simplex virus and atherosclerosis. Eur Heart J 1993;Suppl K:39-42. 